\title{
Cement Leakage in Percutaneous Vertebroplasty for Multiple Osteoporotic Vertebral Compression Fractures: A Prospective Cohort Study
}

This article was published in the following Dove Press journal: Orthopedic Research and Reviews

\author{
Tran Anh Tuan' \\ Tran Van Luong (D) ${ }^{\prime}$ \\ Pham Manh Cuong' \\ $\mathrm{Vu}$ Long ${ }^{2}$ \\ Huynh Quang Huy $\mathbb{D}^{1,3}$ \\ Nguyen Minh Duc (D) ${ }^{1,3}$ \\ 'Department of Radiology, Bach Mai \\ Hospital, Ha Noi, Vietnam; ${ }^{2}$ Department \\ of Radiology, Viet Duc Hospital, Ha Noi, \\ Vietnam; ${ }^{3}$ Department of Radiology, \\ Pham Ngoc Thach University of \\ Medicine, Ho Chi Minh City, Vietnam
}

Correspondence: Huynh Quang Huy Department of Radiology, Pham Ngoc Thach University of Medicine, 2 Duong Quang Trung Street, District 10, Ho Chi Minh City, Vietnam

Tel +84982108108

Email huyhq@pnt.edu.vn
Purpose: This study aims to explore cement leakage as a complication of percutaneous vertebroplasty (PVP) in the treatment of multiple osteoporotic vertebral compression fractures (MOVF).

Patients and Methods: This prospective study was carried out on 32 consecutive patients with osteoporotic fractures of at least two vertebrae (VB). All patients were over 50 years old and women accounted for 29 out of the 32 patients (90.6\%). PVP was performed under digital subtraction angiography (DSA) of at least three VB, and 97 collapsed VB and $105 \mathrm{VB}$ were examined by PVP. All patients had postoperative computerized tomography (CT) to diagnose and classify the complications.

Results: One hundred and five vertebrae were examined with PVP, and 36/105 (34.3\%) exhibited complications of cement leakage. Type B cement leakage was the most common complication, with 19/105 (18.1\%) cases; type C accounted for 8/105 (7.6\%) cases; and type $\mathrm{S}$ accounted for $9 / 105(8.6 \%)$ cases. There was only one $(0.95 \%)$ case of cement leakage moving to the pulmonary artery. All complications had no clinical symptoms and did not require treatment.

Conclusion: Cement leakage is quite a common complication, but it usually has no clinical symptoms and does not require treatment. Therefore, PVP is a safe and successful technique for the treatment of multiple osteoporotic vertebral compression fractures.

Keywords: percutaneous vertebroplasty, PVP, multiple osteoporotic compression fracture, complication, cement leakage, polymethyl methacrylat, PMMA

\section{Introduction}

In the treatment of multiple osteoporotic vertebral compression fractures (MOVF), percutaneous vertebroplasty (PVP) has been shown to be effective in reducing pain and strengthening the vertebral body. ${ }^{1,2}$ PVP is a minimally invasive, non-anesthetic intervention method, with a short intervention duration, effective analgesic, and quick recovery. ${ }^{3-5}$ However, with any intervention, there is a probability of complications. For PVP, cement leakage is one of the most common and important complications. ${ }^{6-8}$

According to Yeom et al., the incidence of cement leakage complications is $38 \%-72.5 \%$. It can lead to pulmonary embolism, neurological deficiency due to spinal cord compression, or peripheral neuropathy due to nerve root damage. ${ }^{9}$

Our study aims to explore cement leakage as a complication of percutaneous vertebroplasty (PVP) in the treatment of multiple osteoporotic vertebral compression fractures (MOVF). 


\section{Patients and Methods}

\section{Patients}

This study was conducted on 32 consecutive patients (29 women and 3 men) with painful osteoporotic compression fractures treated using percutaneous vertebroplasty. The indication for percutaneous vertebroplasty was back pain caused by a vertebral body compression fracture, with pain on percussion of the vertebral spinous process. In some cases, percussion pain of the spinous process was unclear, and a physical examination was performed using fluoroscopy. In addition, patients must be evidence of fresh fracture on magnetic resonance imaging (MRI), osteoporotic vertebral compression fracture can be manifested at various degrees, can be discreetly damaged without changes in vertebrae body height, in which, MRI has high sensitivity and specificity in detecting fresh fracture. Whereby, fresh fracture always has hypointense on T1W, hyperintense on T2W and STIR, so, STIR sequence has the highest sensitivity in detecting bone marrow edema. Moreover, T1W sequence plays an important role in the diagnosis of fracture lines and evaluate morphology of vertebral compression due to $\mathrm{T} 2 \mathrm{~W}$, STIR sequence may obscure fat, fluid and blood organization. Patients with back pain attributed to myelopathy or radiculopathy resulting from stenosis of the vertebral canal or narrowing of the intervertebral foramen were excluded.

Of these 32 patients, there were 5 patients with 2 collapsed vertebrae, 22 patients with 3 collapsed vertebrae, 4 patients with 4 collapsed vertebrae and 1 patient with 5 collapsed vertebrae, so, there were 97 compressed vertebrae. The locations and numbers of 97 collapsed vertebrae (VB) were as follows: $\mathrm{T} 8, \mathrm{n}=2 ; \mathrm{T} 9, \mathrm{n}=5$; $\mathrm{T} 10$, $\mathrm{n}=8 ; \mathrm{T} 11, \mathrm{n}=10 ; \mathrm{T} 12, \mathrm{n}=11 ; \mathrm{L} 1, \mathrm{n}=19 ; \mathrm{L} 2, \mathrm{n}=16 ; \mathrm{L} 3$, $\mathrm{n}=17 ; \mathrm{L} 4, \mathrm{n}=9$. PVP was performed under digital subtraction angiography of at least three $\mathrm{VB}$, some patients with discontinuous multiple vertebral compression fractures (intact vertebrae exist between the fractured vertebrae as discontinuous type), PVP was performed prophylaxis of normal vertebrae between 2 collapsed vertebrae under digital subtraction angiography, so, there were $105 \mathrm{VB}$ performed by PVP.

In accordance with the Declaration of Helsinki, after providing necessary data, a written informed consent was obtained for all patients. The study was approved by the Ethics Committee of Bach Mai hospital under the reference number of 13/2019-BMH.

\section{Percutaneous Vertebroplasty Procedure}

All procedures were performed by a specialist who had more than 10 years of experience in percutaneous vertebroplasty.

We performed all procedures in an angiographic unit with a C-arm digital subtraction angiography (DSA) fluoroscopy system. Patients were kept in a prone position, and all procedures were performed under strict sterile conditions. All procedures were conducted under local anesthesia via a transpedicular approach using a 13 gauge needle. Gentle tapping guided the needle through the pedicle into the anterior third of the involved vertebral body. Frontal and lateral images were recorded with the needle in the correct position.

The prepared polymethyl methacrylat (PMMA) was slowly injected into the vertebral body, and satisfactory infiltration of the vertebral body was confirmed radiographically. The amount of cement injected was variable, depending on the vertebral level that had to be treated and the size of the lesion.

PVP was done with no more than three vertebrae in one intervention, which minimizes the complications associated with cement leakage. ${ }^{10}$ However, consideration should be given to performing PVP for all collapsed vertebrae in one intervention in the case of severe osteoporosis or the longterm use of steroids. ${ }^{5}$ In our study, we aimed to perform PVP

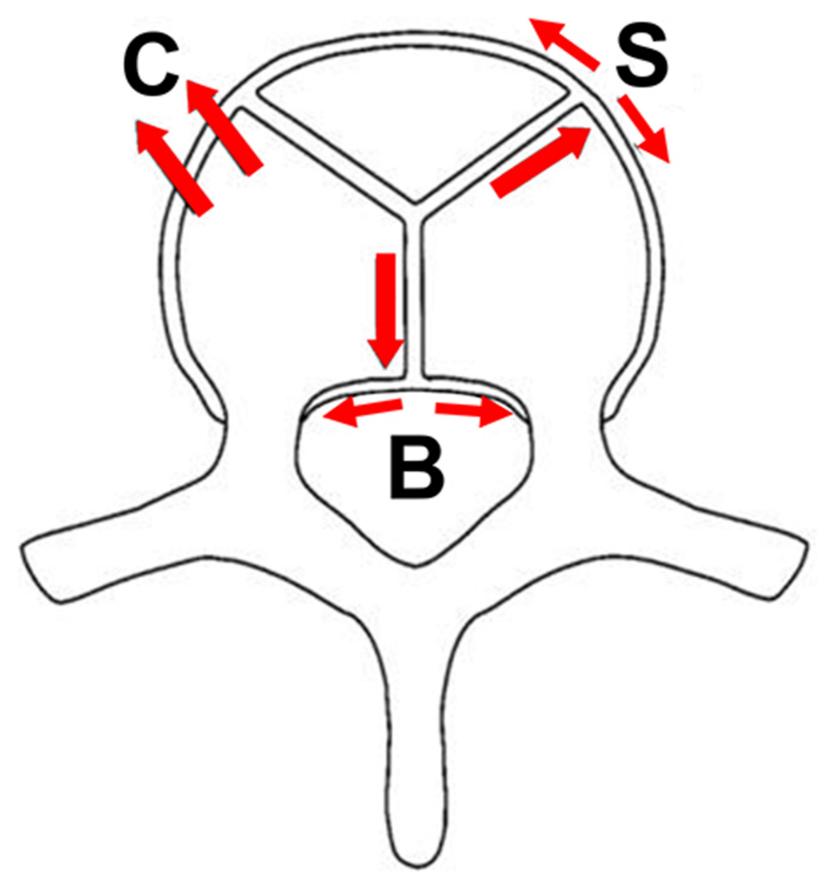

Figure I Types of cement leakage. 
for as many of the collapsed vertebrae as possible, but no more than 5 .

The needle was then removed, and all patients were observed in the supine position for $2 \mathrm{~h}$ after the procedure.

\section{Follow-Up}

After the procedure, patients were completely rested in the first 6 hours and moved gently after 24 hours. All participants in the vertebroplasty group received oral antibiotics for three days and analgesics if needed.

A computerized tomography (CT) scan was performed after the procedure with a Siemens 128-slice machine, reconstructed $3 \mathrm{~mm}$ thin to assess the complication: the

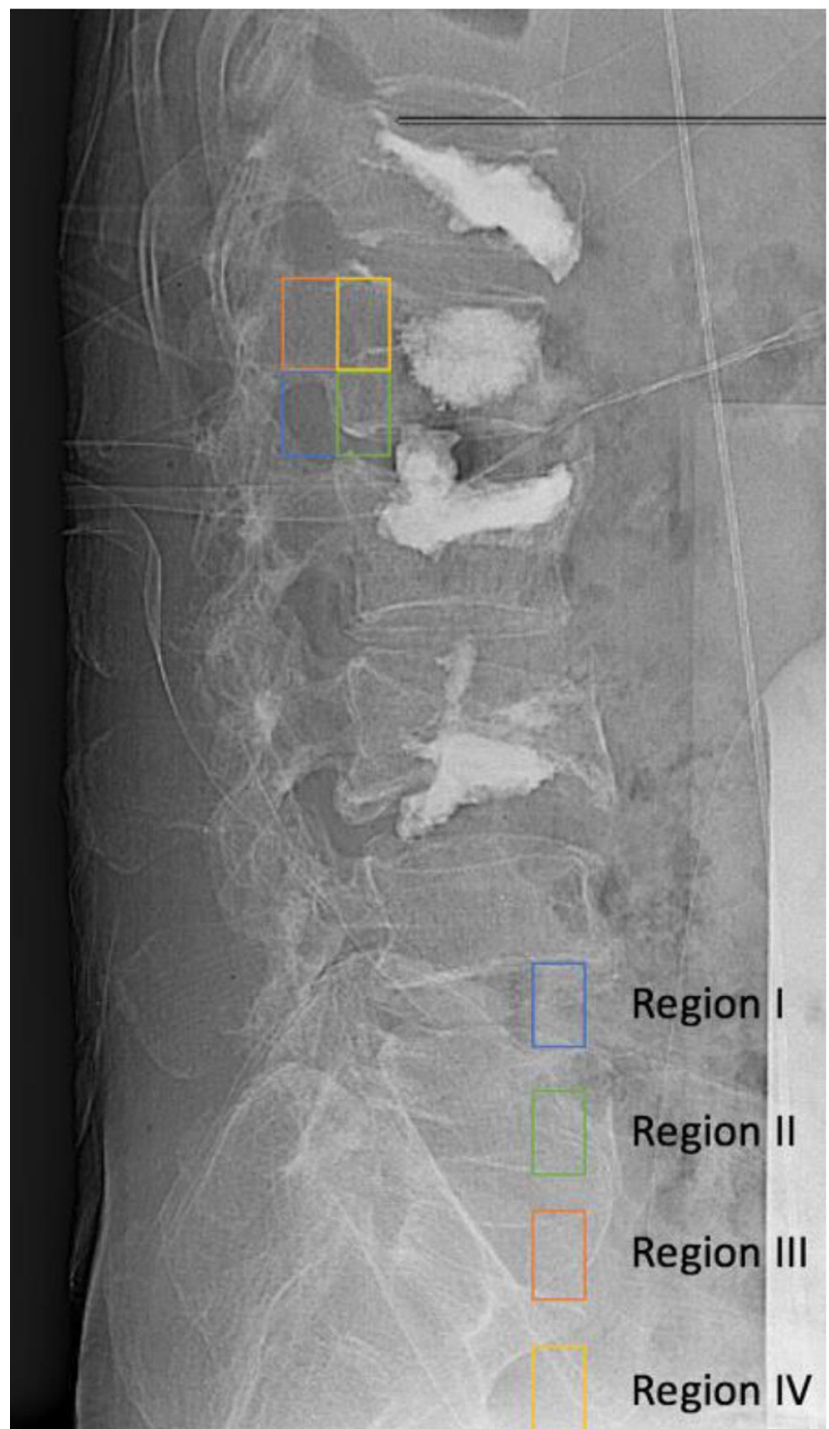

Figure 2 Four anatomical areas of spine on the vertical plane: Region I: the foraminal region; Region II: just prior to the foramen and I/5 of the posterior wall of the vertebral body; Region III: the peduncle region; Region IV: just prior to the peduncle and $1 / 5$ of the posterior wall of the vertebral body.
Table I General Features

\begin{tabular}{|l|l|}
\hline Variables & All Patients $(\mathbf{n}=\mathbf{3 2})$ \\
\hline Age (mean) & $71.6 \pm 9.9$ years (age range, 54-87) \\
Gender (female) & $29(90.6 \%)$ \\
Onset (medium) & 7 days (range, 2-180) \\
VAS score (mean) & 8.25 (range, 3-10) \\
Type of vertebrae & Thorax, Lumbar \\
\hline
\end{tabular}

presence of cement (with contrast material) in structures outside the vertebral body. Complications of cement leakage were divided into three types: ${ }^{9}$ type B represents cement leakage into the basivertebral vein; type $\mathrm{C}$ represents leakage through the cortical defect, including the intervertebral disc, surrounding soft tissue, epidural space, and foramen; and type $\mathrm{S}$ represents leakage into the segmental vein (as shown in Figure 1).

To detect and control complications during the PVP process, we divided the anatomy of the spine into four areas (on the vertical plane): Region I: the foraminal region; Region II: just prior to the foramen and 1/5 of the posterior wall of the vertebral body; Region III: the peduncle region; Region IV: just prior to the peduncle and $1 / 5$ of the posterior wall of the vertebral body (Figure 2). Thereby, both of regions III and IV were considered to be the area around the pedicle.

\section{Statistical Analysis}

The data were analyzed using the SPSS statistical analysis program for Windows, version 20. We calculated the

Table 2 Type of Complication (According to the CT Scan PostProcedure)

\begin{tabular}{|l|l|}
\hline Type of Complication & All Treated Vertebra (n = I05) \\
\hline No complication & $69(65.7 \%)$ \\
Type B & $19(18.1 \%)$ \\
Type C & $8(7.6 \%)$ \\
Type S & $9(8.6 \%)$ \\
\hline
\end{tabular}

Table 3 Type of Complication Associated with the Anatomical Region

\begin{tabular}{|l|l|l|l|l|l|}
\hline Region & $\begin{array}{l}\text { Type } \\
\text { B }\end{array}$ & $\begin{array}{l}\text { Type } \\
\text { C }\end{array}$ & $\begin{array}{l}\text { Type } \\
\text { S }\end{array}$ & $\begin{array}{l}\text { No } \\
\text { Complication }\end{array}$ & Total \\
\hline I & 0 & 0 & 0 & 0 & 0 \\
II & 0 & 0 & 0 & I & I \\
III & 5 & 0 & 0 & 2 & 7 \\
IV & I2 & 0 & I & 13 & 26 \\
\hline
\end{tabular}


frequencies of the categorical variables and the means of the continuous variables.

\section{Result}

Thirty-two patients with osteoporotic fractures of at least two vertebrae (VB) fulfilled the inclusion criteria. The mean patient age was $71.6 \pm 9.9$ years (age range, 54-87 years). The medium onset was 7 days (range, 2-180) and the mean VAS score was 8.25 (range, 3-10) (as shown in Table 1).
One hundred and five vertebrae were examined with PVP, and 36/105 (34.3\%) exhibited complications of cement leakage. Type B cement leakage was the most common complication, with 19/105 (18.1\%) cases; type $\mathrm{C}$ represented 8/105 (7.6\%) cases; and type S accounted for $9 / 105(8.6 \%)$ cases. There was only one $(0.95 \%)$ case of cement leakage moving into the pulmonary artery (Table 2). Of the seven lesions in region III, 5/7 (71.4\%) were type B complications, and positive predictive values (PPV) accounted for $71.4 \%$. Of the 26 lesions in region IV,

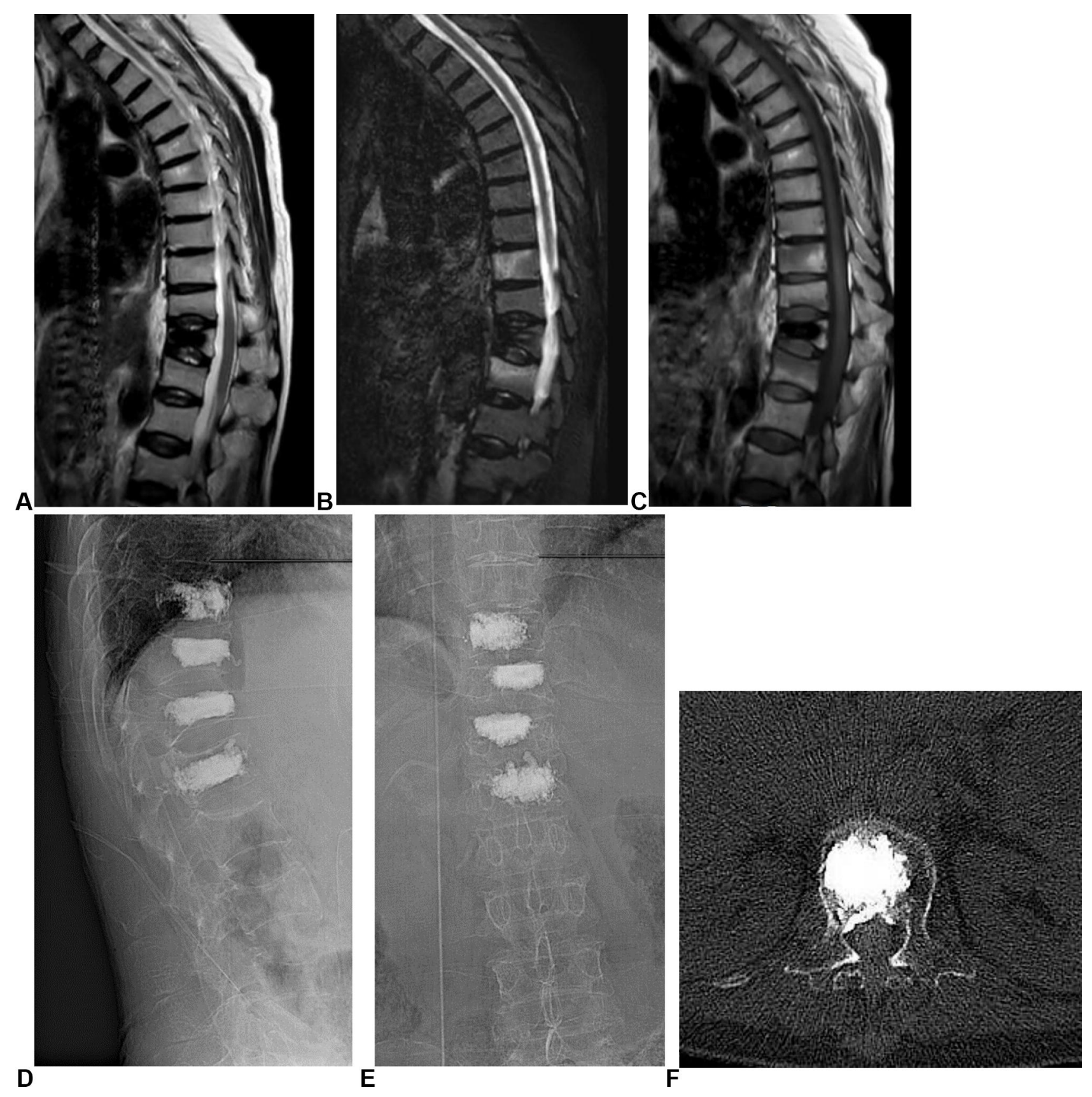

Figure 3 The imaging of a 60-year-old female patient. (A-C) MRI pre-procedure. (D and E) Images post-procedure. (F) Type B complication. 
12/26 were type B complications, and PPV accounted for $46.1 \%$ (Table 3 ).

\section{Discussion}

MOVF is a common medical problem and a burden to the health system. In particular, the compression of vertebrae is considered a consequence of osteoporosis due to the reduced bone density and thin cortical shell. The main clinical symptom is pain. Depending on the degree of pain, this may result in a decrease in motor function and the quality of life of the patient.

According to Tseng et al., each compressed vertebra due to osteoporosis will increase the risk of compression of another vertebra by $20 \%{ }^{11}$

PVP in the treatment of MOVF is an effective treatment method, and quickly improves the symptoms of pain, the motor function of patients, and the quality of life. This is a minimally invasive, fast, and relatively safe treatment. However, complications associated with intervention are inevitable.

For complications of PVP, Nizar A. et al divided them into three groups. Mild complications are transient, requiring no treatment and leaving no sequelae. These include increased pain after the procedure, transient hypotension, and cement leakage into the intervertebral disc or soft tissue. Moderate complications need to be treated, including infectious cement leakage into the epidural space or the foramen. Severe complications consist of a group of potentially fatal complications, including cement leakage into the segmental vein, which can lead to pulmonary embolism, cerebral infarction, or heart perforation. ${ }^{6}$

Cement leakage is a common complication in PVP, and cement leakage into the basivertebral vein (type B) is the most common type. In our study, type B complications accounted for 18.1\% (19/105) of cases (Table 2). However, all cases with type B complications were asymptomatic. Cement leakage into the basivertebral plexus can cause spinal stenosis. The cement is distributed widely along the plexus, so this condition may be considered a common localized venous stagnation condition without symptoms of compression. ${ }^{9}$ In terms of the images, the distribution of cement in the basivertebral vein is symmetrical on both sides, the cement lies in front of the spinal cord, along the posterior wall, and there is no displacement of more than $1 / 3$ of the diameter of the spinal cavity (Figure 3).

Type C complications accounted for $7.6 \%$ (8/105) of cases. The cement leakage that occurred through the cortical defect, including cement leakage into the intervertebral disc (Figure 4), epidural space (needs to be distinguished from type B), foramen, and soft tissue. Compared with type $\mathrm{B}$ complications, type $\mathrm{C}$ complications often have a worse prognosis and may cause clinical symptoms of compression. Anatomically, in type B complications, the cement is usually limited by the epidural plexus, whilst type $\mathrm{C}$ complications have no protective barrier. Therefore, the cement can easily penetrate the epidural space, causing severe spinal stenosis, and the invasion of nerve roots or surrounding blood vessels, which can lead to a decreased motor or sensory function.
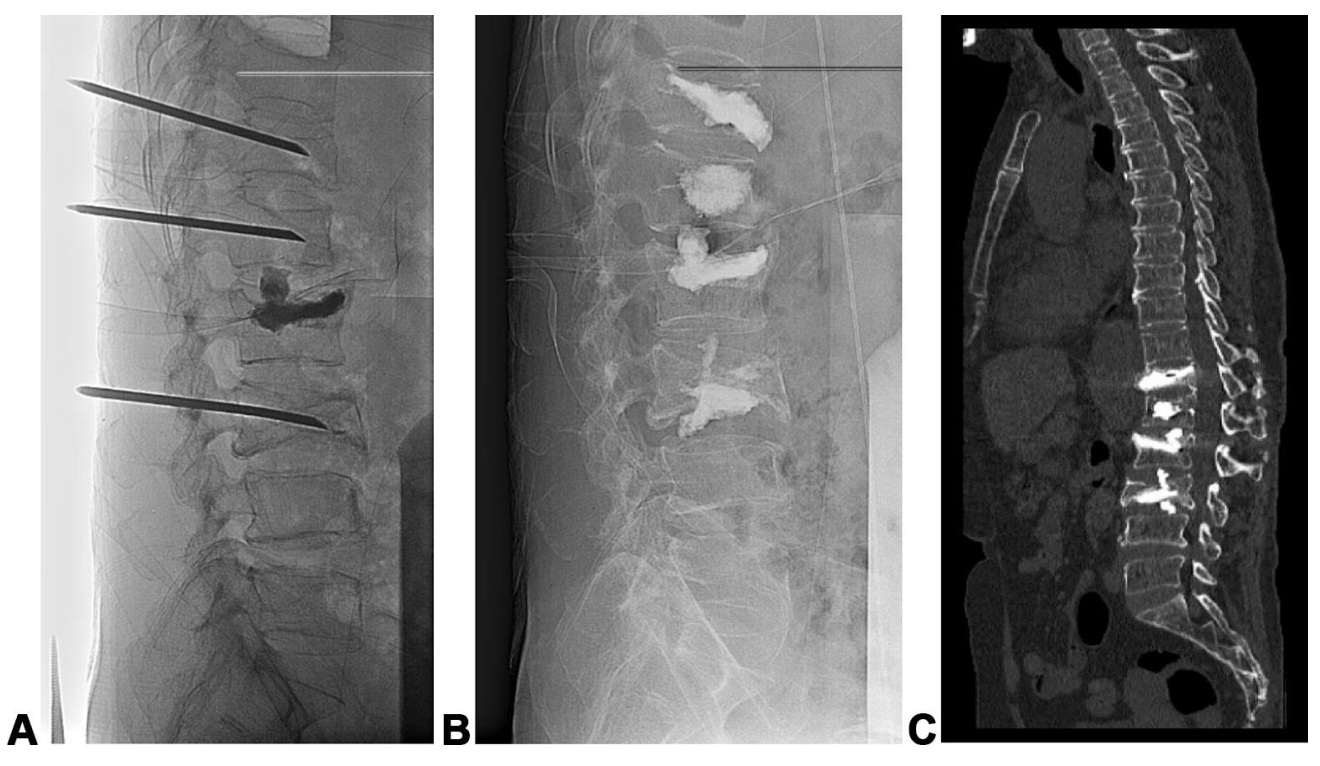

Figure 4 The imaging of a 58-year-old female patient. (A) During the procedure. (B) Post-procedure. (C) Type C complication (L3). 


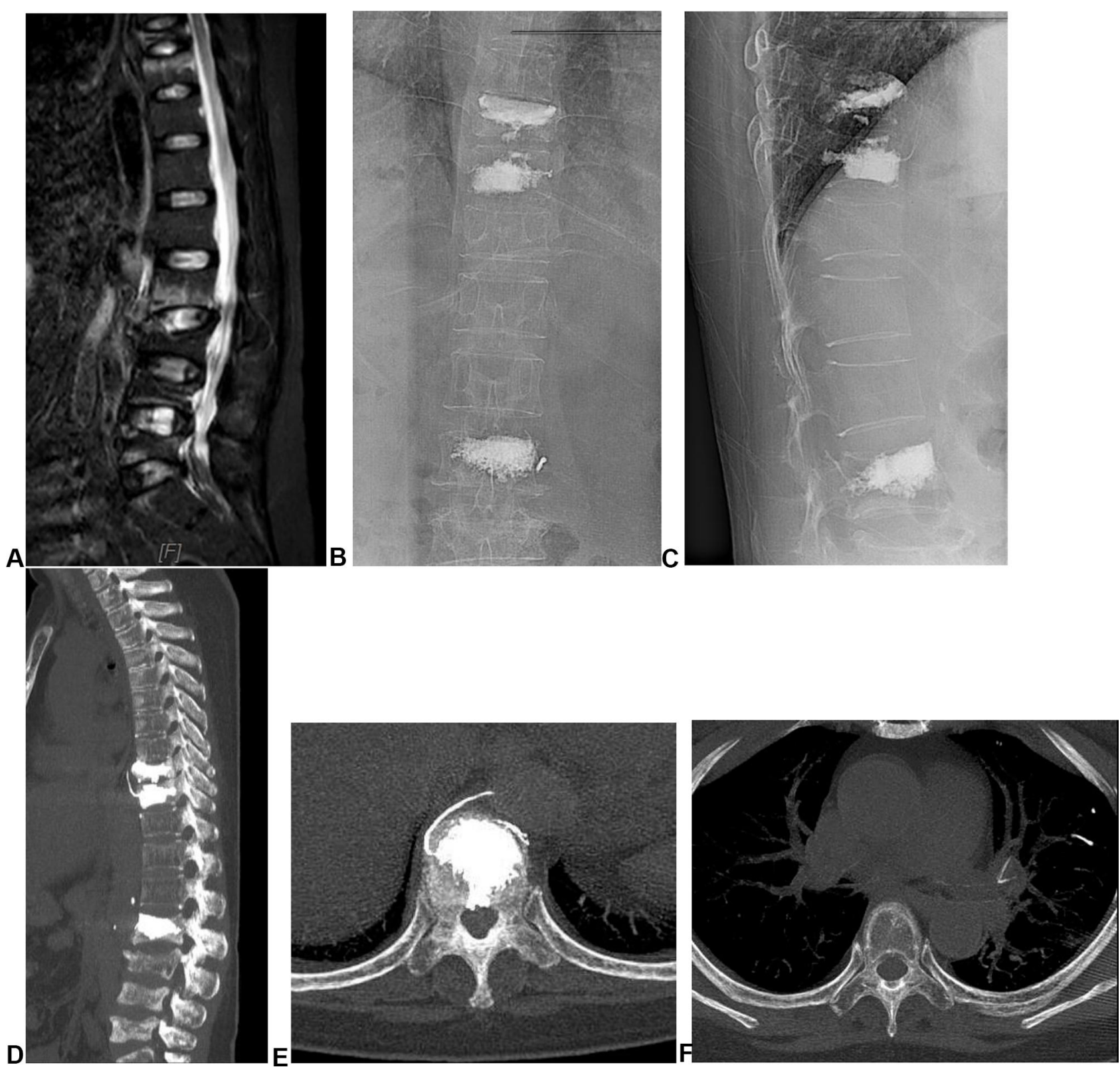

Figure 5 The imaging of a 57-year-old female patient: (A) MRI of the spine: abnormal T9, I0, and II. (B and C): post-percutaneous vertebroplasty (PVP). (D and E) type S complication. (F) pulmonary embolism.

Therefore, type $\mathrm{C}$ is considered to be much more dangerous than type $\mathrm{B}$. The distribution of cement in type $\mathrm{C}$ is usually asymmetrical, displaces more than $1 / 3$ of the diameter of the spinal cavity, and causes severe spinal stenosis. In particular, there are two types of fracture that can lead to type $\mathrm{C}$ complications. Firstly, fractures caused by osteoporosis, especially in the acute phase. Secondly, the cortical defect due to the needle's path. In our study, type C complications were observed in 8/105 (7.6\%) cases. All are complications that consist of cement leakage into the intervertebral disc. Many studies have argued that this is one of the risk factors for new compressed vertebra. Cement leakage into the disc has been shown to lead to a loss of buffer system properties, reduced elasticity, and force dispersion. ${ }^{8}$ In order to minimize the risk of cement leakage into the disc, the first thing one has to pay attention to is the fact that the needle tip should not be located too close to the center of the vertebra. Secondly, the cement needs to be mixed to increase the viscosity and reduce the excessive diffusion. In addition, the volume of cement pumped should be as small as possible to not only create a therapeutic effect but also reduce the risk of cement leakage. A minimum cement volume of $1.5 \mathrm{~mL}$ is sufficient for creating an analgesic 
effect. ${ }^{8}$ Last but not least, the procedure should be stopped immediately when signs of complications are observed.

Type S complications were found in 9/105 cases, accounting for $8.6 \%$ (Table 2). Type $\mathrm{S}$ complications include the presence of the segmental vein (Figure 5). Type $\mathrm{S}$ is considered as severe complications, which are potentially fatal. The cement can move along the veins to the heart, causing heart perforation, pulmonary embolism, and cerebral infarction. In our study, one in 105 of cases $(0.95 \%)$ reported the presence of cement in the pulmonary artery; however, this patient showed no chest pain, shortness of breath, or damage to surrounding lung parenchyma. This condition may have been due to the occlusion of small branches. The main symptoms of pulmonary embolism are chest pain, shortness of breath, and hypotension. ${ }^{8}$ Onset may be immediate or delayed, and can lead to death. Prophylactic treatment includes oxygen, shortterm anticoagulation, intravenous steroid therapy, and steroid therapy with a central intravenous catheter in severe cases.

To detect complications early, we divided the spine into four anatomical regions on the vertical plane. Each region had different predictive values for cement leakage complications (Table 3). The presence of cement mainly occurred in region III and IV, possibly because the needle path we chose went through the peduncle. Of the seven lesions in region III, 5/7 (71.4\%) were type B complications, and positive predictive values (PPV) accounted for $71.4 \%$. Of the 26 lesions in region IV, 12/26 were type B complications, and PPV accounted for $46.1 \%$. According to Yeom et al., ${ }^{9}$ the presence of cement in region I and II indicates a worse prognosis due to the risk of causing severe spinal stenosis and foraminal compression.

\section{Conclusion}

Complications associated with cement leakage in PVP treatment are common but usually have no clinical symptoms and do not require treatment. In this study, the presence of cement in the pulmonary artery was observed in one of 105 cases. This case was asymptomatic due to the occlusion of small branches.

\section{Funding}

This work did not receive any funding.

\section{Disclosure}

The authors report no conflicts of interest in this work.

\section{References}

1. Stevenson M, Gomersall T, Lloyd Jones M, et al. Percutaneous vertebroplasty and percutaneous balloon kyphoplasty for the treatment of osteoporotic vertebral fractures: a systematic review and cost-effectiveness analysis. Health Technol Assess (Rockv). 2014;18 (17):1-290. doi:10.3310/hta18170

2. Widmer Soyka RP, Helgason B, Hazrati Marangalou J, van den Bergh JP, van Rietbergen B, Ferguson SJ. The effectiveness of percutaneous vertebroplasty is determined by the patient-specific bone condition and the treatment strategy. PLoS One. 2016;11(4): e0151680. doi:10.1371/journal.pone. 0151680

3. Alexandru D, So W. Evaluation and management of vertebral compression fractures. Perm J. 2012;16(4):46-51. doi:10.7812/TPP/12037

4. Anselmetti GC, Corrao G, Monica PD, et al. Pain relief following percutaneous vertebroplasty: results of a series of 283 consecutive patients treated in a single institution. Cardiovasc Intervent Radiol. 2007;30(3):441-447. doi:10.1007/s00270-006-0146-0

5. Guarnieri G, Masala S, Muto M. Update of vertebral cementoplasty in porotic patients. Interv Neuroradiol. 2015;21(3):372-380. doi:10.1177/1591019915582364

6. Al-Nakshabandi NA. Percutaneous vertebroplasty complications. Ann Saudi Med. 2011;31(3):294-297. doi:10.4103/0256-4947.81542

7. Cosar M, Sasani M, Oktenoglu T, et al. The major complications of transpedicular vertebroplasty. $J$ Neurosurg Spine. 2009;11 (5):607-613. doi:10.3171/2009.4.SPINE08466

8. Gangi A, Guth S, Imbert JP, Marin H, Dietemann JL. Percutaneous vertebroplasty: indications, technique, and results. Radiographics. 2003;23(2):e10. doi:10.1148/rg.e10

9. Yeom JS, Kim WJ, Choy WS, Lee CK, Chang BS, Kang JW. Leakage of cement in percutaneous transpedicular vertebroplasty for painful osteoporotic compression fractures. J Bone Joint Surg. 2003;85(1):83-89. doi:10.1302/0301-620X.85B1.13026

10. Yu SW, Yang SC, Kao YH, Yen CY, Tu YK, Chen LH. Clinical evaluation of vertebroplasty for multiple-level osteoporotic spinal compression fracture in the elderly. Arch Orthop Trauma Surg. 2008;128(1):97-101. doi:10.1007/s00402-007-0426-4

11. Tseng YY, Yang TC, Tu PH, Lo YL, Yang ST. Repeated and multiple new vertebral compression fractures after percutaneous transpedicular vertebroplasty. Spine. 2009;34(18):1917-1922. doi:10.1097/ BRS.0b013e3181ac8f07
Orthopedic Research and Reviews

\section{Publish your work in this journal}

Orthopedic Research and Reviews is an international, peer-reviewed, open access journal that focusing on the patho-physiology of the musculoskeletal system, trauma, surgery and other corrective interventions to restore mobility and function. Advances in new technologies, materials, techniques and pharmacological agents are particularly welcome. The manuscript management system is completely online and includes a very quick and fair peer-review system, which is all easy to use. Visit http://www.dovepress.com/testimonials.php to read real quotes from published authors. 